PROCEEDINGS OF THE

AMERICAN MATHEMATICAL SOCIETY

Volume 132, Number 8, Pages 2221-2231

S 0002-9939(04)07478-7

Article electronically published on March 4, 2004

\title{
A GENERALIZATION OF A THEOREM OF RANKIN AND SWINNERTON-DYER ON ZEROS OF MODULAR FORMS
}

\author{
JAYCE GETZ
}

(Communicated by David E. Rohrlich)

\begin{abstract}
Rankin and Swinnerton-Dyer (1970) prove that all zeros of the Eisenstein series $E_{k}$ in the standard fundamental domain for $\Gamma$ lie on $A:=$ $\left\{e^{i \theta}: \frac{\pi}{2} \leq \theta \leq \frac{2 \pi}{3}\right\}$. In this paper we generalize their theorem, providing conditions under which the zeros of other modular forms lie only on the arc $A$. Using this result we prove a speculation of Ono, namely that the zeros of the unique "gap function" in $M_{k}$, the modular form with the maximal number of consecutive zero coefficients in its $q$-expansion following the constant 1 , has zeros only on $A$. In addition, we show that the $j$-invariant maps these zeros to totally real algebraic integers of degree bounded by a simple function of weight $k$.
\end{abstract}

\section{Introduction AND STATEMENT OF RESUlts}

Let $\mathbb{H}$ denote the complex upper half-plane and $\Gamma:=\mathrm{SL}_{2}(\mathbb{Z})$. The region

$$
\mathbf{F}:=\left\{|z| \geq 1 \text { and }-\frac{1}{2} \leq \operatorname{Re}(z) \leq 0\right\} \bigcup\left\{|z|>1 \text { and } 0 \leq \operatorname{Re}(z)<\frac{1}{2}\right\}
$$

is the usual fundamental domain for $\mathbb{H}$ under the action of $\Gamma$. That is, $\mathbf{F}$ serves as a set of representatives for equivalence classes for $\mathbb{H}$ under the action of $\Gamma$ by fractional linear transformations. We say that a meromorphic function $f$ on $\mathbb{H}$ is a modular function of weight $k$ for $\Gamma$ if

$$
f\left(\frac{a z+b}{c z+d}\right)=(c z+d)^{-k} f(z)
$$

for all $z \in \mathbb{H}$ and $\left(\begin{array}{ll}a & b \\ c & d\end{array}\right) \in \Gamma$. Furthermore, if $f$ is holomorphic on $\mathbb{H}$ and at the cusps of $\mathbf{F}$, it is a modular form. Denote by $M_{k}$ the finite-dimensional complex vector space of such modular forms under the action of $\Gamma$. The description of the zeros of a modular function $f \in M_{k}$ on $\mathbb{H}$ is clearly equivalent to the description of the zeros of $f$ on $\mathbf{F}$. Thus, for the remainder of this paper, when we speak of a zero $z_{0}$ of $f \in M_{k}$, we assume $z_{0} \in \mathbf{F}$.

Received by the editors March 21, 2003.

2000 Mathematics Subject Classification. Primary 11F11.

Key words and phrases. Modular forms.

The author thanks the University of Wisconsin at Madison for its support.

(C)2004 American Mathematical Society 
For even integers $k \geq 2$, let $E_{k}(z)$ be the usual Eisenstein series

$$
E_{k}(z):=1-\frac{2 k}{B_{k}} \sum_{n=1}^{\infty} \sigma_{k-1}(n) q^{n},
$$

where $\sigma_{k-1}(n)=\sum_{d \mid n} d^{k-1}$ and $B_{k}$ is the $k$ th Bernoulli number. Throughout this paper, $q=e^{2 \pi i z}$ and we make the convention that $E_{0}(z):=1$. Recall that if $k \geq 4$ is even, then $E_{k}(z) \in M_{k}$. In [RSD, Rankin and Swinnerton-Dyer prove that if $E_{k}(z)=0$ and $z \in \mathbf{F}$, then $z \in A$, where

$$
A:=\left\{e^{i \theta}: \frac{\pi}{2} \leq \theta \leq \frac{2 \pi}{3}\right\} .
$$

In this paper we generalize their result, providing a method of determining that the zeros of a modular form $f$ on $\mathbf{F}$ lie only on the arc $A$. Before we state this result, we recall the following definitions:

$$
j(z):=q^{-1}+744+196884 q+\cdots
$$

is the usual normalized weight zero modular function and

$$
\Delta(z)=\frac{E_{4}(z)^{3}-E_{6}(z)^{2}}{1728}=q-24 q^{2}+252 q^{3}-1472 q^{4}+\cdots
$$

is the unique normalized weight 12 cusp form on $\Gamma$. Furthermore, we define

$$
\epsilon:=e^{-\pi \sqrt{3}}\left(\sum_{n=-\infty}^{\infty} e^{-\pi \sqrt{3}\left(3 n^{2}-n\right) / 2}\right)^{24} \sim 0.004809 \ldots
$$

We note here, and will prove later in Proposition 2.2, that $|\Delta(z)| \leq \epsilon$ for $z \in A$. Finally, if $k=12 m+s$, where $s=0,4,6,8,10,14$, let

$$
m(k):=m \text {. }
$$

Theorem 1. Let $k \geq 4$ be even and $f(z)=E_{k}(z)+\sum_{i=1}^{m(k)} a_{i} E_{k-12 i} \Delta^{i} \in M_{k}$, where $a_{i} \in \mathbb{R}$. Suppose

$$
\sum_{i=1}^{m(k)}\left|a_{i}\right| \epsilon^{i}<\frac{1-\delta}{3+\delta}
$$

where $\delta:=.03562$. If $f(z)=0$ and $z \in \mathbf{F}$, then $z \in A$, and $j(z) \in[0,1728]$. In particular, $f$ has $m(k)$ simple zeros on $\left\{e^{i \theta}: \frac{\pi}{2}<\theta<\frac{2 \pi}{3}\right\}$, and we have the following trivial zeros of $f$ depending on $k$ modulo 12 :

$$
\operatorname{ord}_{i}(f)=\left\{\begin{array}{lll}
1 & \text { if } k \equiv 2 \quad(\bmod 4), \\
0 & \text { if } k \equiv 0 & (\bmod 4),
\end{array}\right.
$$

and

$$
\operatorname{ord}_{\rho}(f)=\left\{\begin{array}{lll}
2 & \text { if } k \equiv 2 & (\bmod 6) \\
1 & \text { if } k \equiv 4 \quad(\bmod 6) \\
0 & \text { if } k \equiv 0 \quad(\bmod 6)
\end{array}\right.
$$

Here $\rho:=e^{2 \pi i / 3}$.

A natural question to ask is what other specific families of modular forms have the property that their zeros lie only on the arc $A$. In Section 3 we provide such a family, the so-called "gap functions". 
Definition. If $k \geq 4$ is even, the gap function $F_{k}(z) \in M_{k}$ is the unique modular form with Fourier expansion

$F_{k}(z)=1+c(m(k)+1) q^{m(k)+1}+c(m(k)+2) q^{m(k)+2}+\cdots=1+\sum_{n \geq m(k)+1} c(n) q^{n}$

where $c(n) \in \mathbb{C}$ is the nth Fourier coefficient.

For example, we have

$$
F_{12}(z)=E_{12}(z)+\frac{24}{B_{12}} \Delta(z)=1+196560 q^{2}+16773120 q^{3}+\cdots .
$$

These and similar functions are useful in coding theory. For example, MOS] considered the parity of the real parts of the coefficients $c(m(k)+1), c(m(k)+2), \ldots$ of $F_{k}$ among other $q$-series. In this work we are interested in the zeros of $F_{k}$. Ono speculated that these zeros of $F_{k}$ all lie on the arc $A$. By applying the method of proof underlying Theorem 1, we prove this result:

Theorem 2. Suppose $k \geq 4$ is even. If $F_{k}(z)=0$ and $z \in \mathbf{F}$, then $z \in A$ and $j(z) \in[0,1728]$, with $m(k)$ simple zeros on the interior of the arc. Moreover, $F_{k}$ has trivial zeros at $i$ and $\rho$ depending on $k$ modulo 12 as in (1.8).

We can construct totally real extensions of $\mathbb{Q}$ by adjoining $j\left(z_{0}\right)$, where $z_{0}$ is a zero of any of the functions described in Theorems 1 and 2 . It is a well-known fact from the theory of complex multiplication that if $z$ is a CM point, then $j(z)$ is an algebraic integer. We observe an analogous phenomenon in the case of the zeros of the gap functions; the $j$-invariant maps them to totally real algebraic integers. In addition, for certain weights, these algebraic integers reduce to supersingular $j$-invariants in $\overline{\mathbb{F}}_{p}$.

Corollary 3. Suppose $k \geq 4$ is even. If $F_{k}(z)=0$ and $z \in \mathbf{F}$, then $j(z)$ is an algebraic integer. If $k=p-1$, where $p \geq 5$ is prime, then there is a maximal ideal $\mathbf{m}$ of the ring of integers of $\mathbb{Q}(j(z))$ lying over $p$ such that $j(z)$ modulo $\mathbf{m}$ is the $j$-invariant of a supersingular elliptic curve.

\section{Preliminaries and proof of Theorem 1}

We begin with a general discussion of the zeros of a nonzero element $f \in M_{k}$. A classical result on this subject is the valence formula (see $\S[$ III.2] $[\mathrm{K}]$ ):

$$
\frac{k}{12}=\frac{1}{2} \operatorname{ord}_{i}(f)+\frac{1}{3} \operatorname{ord}_{\rho}(f)+\operatorname{ord}_{\infty}(f)+\sum_{\tau \in \Gamma \backslash \mathbb{H}-\{i, \rho\}} \operatorname{ord}_{\tau}(f) .
$$

Writing $k=12 m(k)+s$ as in (1.7), note that $s$ determines the residue class of $k$ modulo 12. Bearing in mind the valence formula, an examination of the possible values of $s$ implies that

$$
\operatorname{ord}_{i}(f) \geq\left\{\begin{array}{lll}
1 & \text { if } k \equiv 2 \quad(\bmod 4), \\
0 & \text { if } k \equiv 0 \quad(\bmod 4),
\end{array}\right.
$$

and

$$
\operatorname{ord}_{\rho}(f) \geq\left\{\begin{array}{lll}
2 & \text { if } k \equiv 2 \quad(\bmod 6) \\
1 & \text { if } k \equiv 4 \quad(\bmod 6) \\
0 & \text { if } k \equiv 0 & (\bmod 6)
\end{array}\right.
$$


Again applying the valence formula for $k=12 m(k)+s$, by $(2.1)$, there are at most $m(k)$ zeros on $\mathbf{F}-\{\rho, i\}$. Thus if $f \in M_{k}$ satisfies the hypotheses of Theorem 1 , then to prove Theorem 1 it suffices to demonstrate that $f$ has $m(k)$ simple zeros in the interior of $A$.

We now require a proposition on normalization of modular functions on the arc $A$.

Proposition 2.1. If $f$ is a modular function of weight $k$ with real coefficients, then $e^{i k \theta / 2} f\left(e^{i \theta}\right)$ is real on $\left\{\theta: \frac{\pi}{2} \leq \theta \leq \frac{2 \pi}{3}\right\}$.

Proof. From the functional equation (1.1) we have $f(-1 / z)=z^{k} f(z)$. For $z=$ $a+b i \in A$, we have $-\frac{1}{z}=\frac{-a+b i}{a^{2}+b^{2}}=-a+b i$. Write $f$ in terms of its Fourier expansion as

$$
f(z)=\sum_{n \geq n_{f}} a_{n} e^{2 \pi i n(a+b i)}=\sum_{n \geq n_{f}} a_{n} e^{2 \pi n(-b+a i)},
$$

where $n_{f}$ is an integer that depends on $f$. We have

$$
f(-1 / z)=\sum_{n \geq n_{f}} a_{n} e^{2 \pi i n(-a+b i)}=\sum_{n \geq n_{f}} a_{n} e^{2 \pi n(-b-a i)}=\overline{f(z)} .
$$

Note that $-1 / e^{i \theta}=e^{i(\pi-\theta)}$. The above facts imply that

$$
\begin{aligned}
e^{i k(\pi-\theta) / 2} f\left(e^{i(\pi-\theta)}\right) & =e^{i k(\pi-\theta) / 2} e^{i k \theta} f\left(e^{i \theta}\right), \\
e^{i k(\pi-\theta) / 2} \overline{f\left(e^{i \theta}\right)} & =e^{i k(\pi+\theta) / 2} f\left(e^{i \theta}\right), \\
e^{-i k \theta / 2} \overline{f\left(e^{i \theta}\right)} & =e^{i k \theta / 2} f\left(e^{i \theta}\right), \\
\overline{e^{i k \theta / 2} f\left(e^{i \theta}\right)} & =e^{i k \theta / 2} f\left(e^{i \theta}\right) .
\end{aligned}
$$

Notice that Proposition 2.1 shows us that $j(z)$ is real for $z \in A$. Furthermore, we have that $j(i)=1728, j(\rho)=0$, and that $j$ is a bijection between $\mathbf{F}$ and $\mathbb{C}$. An application of the intermediate value theorem implies that $j(z) \in[0,1728]$ for all $z \in A$. Similarly, in [RSD], Rankin and Swinnerton-Dyer use the intermediate value theorem to describe the zeros of $E_{k}(z)$ for $k \geq 12$ after proving that

$$
e^{i k \theta / 2} E_{k}\left(e^{i \theta}\right)=2 \cos (k \theta / 2)+R_{k},
$$

where

$$
\left|R_{k}\right|<1+\delta
$$

and where $\delta:=.03562$ as above. A stronger bound will not be necessary for the purposes of this paper, though one could be easily calculated (again, see [RSD]). However, we will need a bound for $\Delta(z)$ when $z \in A$, which we now provide:

Proposition 2.2. If $z \in A$, then $|\Delta(z)| \leq \epsilon$, where $\epsilon$ is defined as in (1.6).

Proof. A standard identity (see $\S[$ III.2] $[\mathrm{K}]$ ) gives us

$$
\Delta(z)=e^{2 \pi i z} \prod_{n=1}^{\infty}\left(1-e^{2 \pi i n z}\right)^{24}=\left(e^{2 \pi i z / 24} \prod_{n=1}^{\infty}\left(1-e^{2 \pi i n z}\right)\right)^{24} .
$$


By Euler's pentagonal number theorem $\S[14.5][\mathrm{A}]$, we have

$$
\prod_{n=1}^{\infty}\left(1-e^{2 \pi i n z}\right)=\sum_{n=-\infty}^{\infty}(-1)^{n} e^{2 \pi i z\left(3 n^{2}-n\right) / 2} .
$$

Writing $z=e^{i \theta}=a+b i$ for $a, b \in \mathbb{R}$, we have

$$
\left|\prod_{n=1}^{\infty}\left(1-e^{2 \pi i n z}\right)\right| \leq \sum_{n=-\infty}^{\infty}\left|(-1)^{n} e^{2 \pi i(a+b i)}\right|^{\left(3 n^{2}-n\right) / 2}=\sum_{n=-\infty}^{\infty} e^{-\pi b\left(3 n^{2}-n\right)} .
$$

Now, for $z=a+b i \in A, e^{-\pi b\left(3 n^{2}-n\right)}$ is maximized at $z=e^{2 \pi i / 3}=-\frac{1}{2}+\frac{\sqrt{3}}{2} i$. So

$$
\left|e^{2 \pi i z}\right|\left|\prod_{n=1}^{\infty}\left(1-e^{2 \pi i n z}\right)\right|^{24} \leq e^{-\pi \sqrt{3}}\left(\sum_{n=-\infty}^{\infty} e^{-\pi \sqrt{3}\left(3 n^{2}-n\right) / 2}\right)^{24}=\epsilon .
$$

A MAPLE calculation shows that $\epsilon \sim 0.004809 \ldots$

We are now ready to proceed with the proof of the main theorem.

Proof of Theorem 1. Write $k=12 m(k)+s$ as in (1.7). The theorem is easily proven for the lower weights by noting that $E_{4}$ and $E_{6}$ are supported by zeros at $\rho$ and $i$ respectively, and that for $k<12$ and $k=14$, we have $E_{k}=E_{4}^{a} E_{6}^{b}$, where $a, b \in \mathbb{Z}_{\geq 0}$ are chosen so that $4 a+6 b=k \S[$ III.2] [K]. Now suppose that $k=12$ or $k>14$. Write

$$
H(\theta):=e^{i k \theta / 2} f\left(e^{i \theta}\right)=H_{0}(\theta)+\sum_{j=1}^{m(k)} a_{j} e^{12 j i \theta / 2} \Delta^{j} H_{j}(\theta),
$$

where $H_{j}(\theta)=e^{(k-12 j) i \theta / 2} E_{k-12 j}\left(e^{i \theta}\right)$. By $(2.2)$ and $(2.3)$, we have $H_{j}(\theta)=$ $2 \cos ((k-12 j) \theta / 2)+R_{k-12 j}$, where $\left|R_{k-12 j}\right|<1+\delta$. Thus

$$
H(\theta):=2 \cos (k \theta / 2)+R_{k}+\sum_{j=1}^{m(k)} a_{j} e^{12 j i \theta / 2} \Delta^{j}\left(2 \cos ((k-12 j) \theta / 2)+R_{k-12 j}\right) .
$$

The bound on $\Delta(z)$ from Proposition 2.1, (2.2) and (2.3) imply that

$$
\left|R_{k}+\sum_{j=1}^{m(k)} a_{j} e^{12 j i \theta / 2} \Delta^{j}\left(2 \cos ((k-12 j) \theta / 2)+R_{k-12 j}\right)\right| \leq 1+\delta+\sum_{j=1}^{m(k)}\left|a_{j}\right| \epsilon^{j}(3+\delta) .
$$

Now, assuming

$$
\sum_{i=1}^{m(k)}\left|a_{i}\right| \epsilon^{i}<\frac{1-\delta}{3+\delta}
$$

we have

$$
1+\delta+\sum_{i=1}^{m(k)}\left|a_{i}\right| \epsilon^{i}(3+\delta)<2 .
$$

Let $n$ be an integer such that $\frac{k}{4} \leq n \leq \frac{k}{3}$. Note that $H(\theta)$ is a real-valued function dominated by the trigonometric function $2 \cos (k \theta / 2)$. Considering the bound (2.6), (2.5) implies that $H(2 n \pi / k)$ is strictly positive or negative depending on the parity of $n$. Thus, by the intermediate value theorem, $H(\theta)$ has at least as many zeros in the open interval $(\pi / 2,2 \pi / 3)$ as there are integers in the interval $\left[\frac{k}{4}, \frac{k}{3}\right]$ minus one, 
and this number is $m(k)$. Recalling (2.1) and the following discussion, this fact is sufficient to finish the proof.

\section{Gap Functions and the $j$-InVARiant}

We begin by providing the following bound on the coefficients of the $q$-expansion of arbitrary powers of $\Delta(z)$.

Theorem 3.1. If $s$ is a positive integer, then define integers $\tau_{s}(n)$ by

$$
\Delta(z)^{s}=\sum_{n=0}^{\infty} \tau_{s}(n) q^{n}:=q^{s} \prod_{n=1}^{\infty}\left(1-q^{n}\right)^{24 s} .
$$

If $n \geq 1$, then

$$
\left|\tau_{s}(n)\right| \leq \frac{n^{7 s-1}}{s^{6 s}}
$$

Proof. We have

$$
\tau_{s}(n)=\sum_{\substack{k_{1}+\ldots+k_{s}=n \\ 1 \leq k_{i} \leq n}} \prod_{i=1}^{s} \tau\left(k_{i}\right) .
$$

A well-known result of Deligne (see, for example, $\S[$ III.2] $[\mathrm{K}]$ ) gives the following strong bound on the $q$-expansion coefficients of $\Delta(z)$ :

$$
|\tau(n)| \leq n^{11 / 2} \sigma_{0}(n) .
$$

For $n \geq 40, n^{11 / 2} \sigma_{0}(n) \leq n^{11 / 2}\left(\frac{\log n}{\log 2}+1\right) \leq n^{11 / 2} n^{1 / 2}=n^{6}$, and a simple MAPLE calculation shows that $|\tau(n)| \leq n^{6}$ for $1 \leq n \leq 40$. Thus,

$$
\left|\tau_{s}(n)\right| \leq \sum_{\substack{k_{1}+\ldots+k_{s}=n \\ 1 \leq k_{i} \leq n}} \prod_{i=1}^{s}\left|\tau\left(k_{i}\right)\right| \leq \sum_{\substack{k_{1}+\ldots+k_{s}=n \\ 1 \leq k_{i} \leq n}} \prod_{i=1}^{s} k_{i}^{6},
$$

which implies

$$
\left|\tau_{s}(n)\right| \leq \sum_{\substack{k_{1}+\ldots+k_{s}=n \\ 1 \leq k_{i} \leq n}}\left(\frac{n}{s}\right)^{6 s} \leq n^{s-1}\left(\frac{n}{s}\right)^{6 s} .
$$

Here we use that the maximum product of $s$ positive integers whose sum is $n$ is bounded by $\left(\frac{n}{s}\right)^{s}$. We also use that the first $s-1$ choices $k_{1}, \ldots, k_{s-1}$ determine the choice of $k_{s}$. Thus

$$
\left|\tau_{s}(n)\right| \leq n^{s-1}\left(\frac{n}{s}\right)^{6 s}=\frac{n^{7 s-1}}{s^{6 s}} .
$$

Proof of Theorem 2. We proceed by induction on the weight $k$. Three facts are needed to establish the basis of our induction. For $k \leq 300$, MAPLE calculations, using the method of this proof, establish first that the zeros of $F_{k}$ lie on the arc $A$ as in the statement of the theorem, and second that $\left|e^{i k \theta / 2} F_{k}\left(e^{i \theta}\right)\right|<4$ for $\frac{\pi}{2}<\theta<\frac{2 \pi}{3}$. Write

$$
e^{i k \theta / 2} F_{k}\left(e^{i \theta}\right)=e^{i k \theta / 2} E_{k}\left(e^{i \theta}\right)+\sum_{j=1}^{m(k)} a_{j} e^{12 i j \theta / 2} \Delta\left(e^{i \theta}\right)^{j} e^{(k-12 j) i \theta / 2} F_{k-12 j}\left(e^{i \theta}\right)
$$


with the convention that $F_{0}:=1$. We note here that the $q$-expansion coefficients of $\Delta$ are integers and so $a_{j} \in \mathbb{R}$. The third fact that we require, and will prove later, is that if $k \geq 300$, then

$$
\sum_{j=1}^{m(k)}\left|a_{j}\right| \epsilon^{j}<\frac{1-\delta}{4}
$$

These three facts complete the basis step of our induction. Assuming (3.2), we have

$$
1-\delta>4 \sum_{j=1}^{m(k)}\left|a_{j}\right| \epsilon^{j} \geq \sum_{j=1}^{m(k)}\left|a_{j}\right|\left|e^{12 i j \theta / 2} \Delta^{j}\right|\left|e^{(k-12 j) i \theta / 2} F_{k-12 j}\right|,
$$

which implies

$$
1-\delta>\left|\sum_{j=1}^{m(k)} a_{j} e^{12 i j \theta / 2} \Delta^{j} e^{(k-12 j) i \theta / 2} F_{k-12 j}\right| .
$$

From (3.1), (3.3), (2.2), and (2.3), we have

$$
e^{i k \theta / 2} F_{k}\left(e^{i \theta}\right)=2 \cos (k \theta / 2)+R_{k}+T_{k},
$$

where $\left|R_{k}\right|<1+\delta$ and $\left|T_{k}\right|<1-\delta$. Noting that $\left|R_{k}\right|+\left|T_{k}\right|<2$ and arguing in a manner analogous to the proof of Theorem 1, again assuming (3.2), (3.4) proves that $F_{k}$ has zeros on the $\operatorname{arc} A$ as described in the statement of Theorem 2 . Assuming (3.2) for $k \geq 300$, we must show that $\left|F_{k}(z)\right|<4$ for $z \in A$ to complete the induction. By (2.2), (2.3), and (3.2) we have

$$
\left|e^{i k \theta / 2} F_{k}\left(e^{i \theta}\right)\right|<2+R_{k}+\sum_{j=1}^{m(k)}\left|a_{j}\right|\left|e^{12 i j \theta / 2} \Delta^{j}\right|\left|e^{(k-12 j) i \theta / 2} F_{k-12 j}\right|<4 .
$$

Thus we have proven that $F_{k}$ has zeros on $A$ as desired, and its normalization is bounded in such a way that we can move on to prove the same result for $F_{k+12}$. This completes the induction, though we must now establish (3.2).

As a modular form in $M_{k}, F_{k}=1+c(m(k)+1) q^{m(k)+1}+c(m(k)+2) q^{m(k)+2}+\cdots$ is completely determined by its first $m(k)+1$ coefficients. Thus the problem of calculating the $a_{i}$ can be reduced to a linear algebra problem; given the first few $q$-expansion coefficients, we wish to compute the coefficients $a_{i}$ of the modular form with respect to the alternate basis $E_{k}, \Delta F_{k-12}, \ldots, \Delta^{m(k)} F_{k-12 m(k)}$. Noting that

$$
\Delta^{s} F_{k-12 s}=q^{s}+\tau_{s}(s+1) q^{s+1}+\tau_{s}(s+2) q^{s+2}+\cdots+\tau_{s}(m(k)) q^{m(k)}+\cdots
$$

and applying Cramer's rule, we have

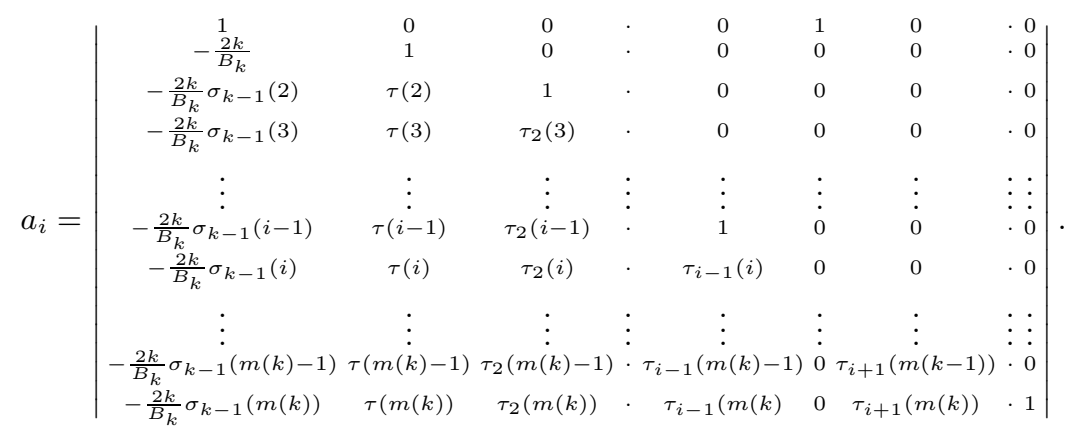


Clearly, $a_{0}=1$ and $a_{1}=\frac{2 k}{B_{k}}$. Fix an $i>1$. To simplify the corresponding matrix, we expand by minors on the $(i+1)$ st column, and then "move up the diagonal", expanding by minors on the $(m(k)+1)$ st column, the $m(k)$ th column, the $(m(k)-1)$ st column, etc., reducing our problem to the calculation of the $i$ by $i$ matrix formed by taking the upper left $(i+1)$ by $(i+1)$ matrix and eliminating the 1 st row and the $(i+1)$ st column. This reduces the calculation of $a_{i}$, up to sign, to the calculation of the determinant of the matrix

$$
\left(\begin{array}{ccccc}
-\frac{2 k}{B_{k}} & 1 & 0 & \cdot & 0 \\
-\frac{2 k}{B_{k}} \sigma_{k-1}(2) & \tau(2) & 1 & \cdot & 0 \\
-\frac{2 k}{B_{k}} \sigma_{k-1}(3) & \tau(3) & \tau_{2}(3) & . & 0 \\
\vdots & \vdots & \vdots & \vdots & \vdots \\
-\frac{2 k}{B_{k}} \sigma_{k-1}(i-1) & \tau(i-1) & \tau_{2}(i-1) & \cdot & 1 \\
-\frac{2 k}{B_{k}} \sigma_{k-1}(i) & \tau(i) & \tau_{2}(i) & \cdot & \tau_{i-1}(i)
\end{array}\right)
$$

Denote the matrix in (3.5) by $D_{k i}=\left(d_{\alpha \beta}\right)$ and note that

$$
\operatorname{det} D_{k i}=\sum_{\sigma \in S_{i}} \operatorname{sgn}(\sigma) d_{1 \sigma(1)} \cdots d_{i \sigma(i)} .
$$

Consider a permutation $\sigma$ of columns of $\left(d_{\alpha \beta}\right)$ that induces a nonzero product $d_{1 \sigma(1)} \cdots d_{i \sigma(i)}$. We have two nonzero choices for $d_{1 \sigma(1)}$. There are three nonzero entries in the 2nd row, and we cannot choose $d_{2 \sigma(1)}$. So there are two nonzero choices. Continuing in this manner and noting that $d_{i \sigma(i)}$ is determined by $d_{1 \sigma(1)}, \ldots$, $d_{i-1 \sigma(i-1)}$, we have $2^{i-1}$ possible nonzero products. With this in mind we define a new function $B$ on the matrices $D_{k i}$ by

$$
B\left(D_{k i}\right)=2^{i-1} \max \left\{\left|d_{1 \sigma(1)}\right| \cdots\left|d_{i \sigma(i)}\right|: \sigma \in S_{i}\right\}
$$

and note that $\left|a_{i}\right|=\left|\operatorname{det} D_{k i}\right| \leq B\left(D_{k i}\right)$.

Now, substituting the trivial bound $\sigma_{k-1}(n) \leq n^{k}$ and the bound of Theorem 3.1 , we have

$$
\left|a_{i}\right| \leq B\left(\begin{array}{ccccc}
\left|\frac{2 k}{B_{k}}\right| & 1 & 0 & \cdot & 0 \\
\left|\frac{2 k}{B_{k}}\right| 2^{k} & 2^{6} & 1 & \cdot & 0 \\
\left|\frac{2 k}{B_{k}}\right| 3^{k} & 3^{6} & \frac{3^{13}}{3^{12}} & \cdot & 0 \\
\vdots & \vdots & \vdots & \vdots & \vdots \\
\left|\frac{2 k}{B_{k}}\right|(i-1)^{k} & (i-1)^{6} & \frac{(i-1)^{13}}{3^{12}} & \cdot & 1 \\
\left|\frac{2 k}{B_{k}}\right| i^{k} & i^{6} & \frac{i^{13}}{3^{12}} & \cdot & \frac{i^{7(i-1)-1}}{(i-1)^{6(i-1)}}
\end{array}\right) .
$$

If we write $D_{i k}$ as a set of $i$ column vectors $\left(v_{1}, \cdots, v_{j}, \cdots, v_{i}\right)$, it follows from the definition of $B$ that $B\left(v_{1}, \cdots, c v_{j}, \cdots, v_{i}\right)=|c| B\left(v_{1}, \cdots, v_{j}, \cdots, v_{i}\right)$ for any complex number $c$. This is because factoring out $|c|$ is equivalent to multiplying every element in the set $\left\{\left|d_{1 \sigma(1)} \cdots d_{i \sigma(i)}\right|: \sigma \in S_{i}\right\}$ by $\left|c^{-1}\right|$, which does not change which element of that set is maximal. Thus factoring $\left|\frac{2 k}{B_{k}}\right|$ from the first column and $\frac{1}{(j-1)^{6(j-1)}}$ from the $j$ th for $j>1$, we have that $(3.6)$ is equal to 
$\left(\left|\frac{B_{k}}{2 k}\right| 2^{12} \cdots(i-1)^{6(i-1)}\right)^{-1}$ times

$$
B\left(\begin{array}{cccccc}
1 & 1 & 0 & \cdots & 0 & 0 \\
2^{k} & 2^{6} & 3^{12} & \cdots & 0 & 0 \\
3^{k} & 3^{6} & 3^{13} & \cdots & 0 & 0 \\
\vdots & \vdots & \vdots & \cdots & \vdots & \vdots \\
(i-1)^{k} & (i-1)^{6} & (i-1)^{13} & \cdots & (i-1)^{7(i-2)-1} & (i-1)^{6(i-1)} \\
i^{k} & i^{6} & i^{13} & \cdots & i^{7(i-2)-1} & i^{7(i-1)-1}
\end{array}\right) .
$$

Denote the matrix in (3.7) by $U_{k i}=\left(u_{\alpha \beta}\right)$. We now claim that

$$
\begin{aligned}
\max \left\{\left|u_{1 \sigma(1)}\right| \cdots\left|u_{i \sigma(i)}\right|: \sigma \in S_{i}\right\} & =u_{12} u_{23} \cdots u_{(i-1) i} u_{i 1} \\
& =i^{k}(1)\left(2^{12}\right) \cdots(i-1)^{6(i-1)} .
\end{aligned}
$$

To see this, first note that we must choose one entry from the first column. Say this entry is $u_{r 1}$. In addition, we must choose one nonzero entry from every row besides the $r$ th, and none of these entries can be in the first column. In order to maximize the product, we want to choose the entry in each row that is not in the first column of maximum absolute value. Thus we choose the entries $u_{12}, u_{23}, \ldots, u_{(r-1) r}, u_{(r+1)(r+2)}, \ldots, u_{(i-1) i}$. Note that the structure of the matrix, which is "almost diagonal", allows this as a possible element of the set $\left\{u_{1 \sigma(1)} \cdots\right.$ $\left.u_{i \sigma(i)}: \sigma \in S_{i}\right\}$. In particular, it gives us the product

$$
u_{12} u_{23} \cdots u_{(r-1) r} u_{r 1} u_{(r+1)(r+2)} \cdots u_{(i-1) i} .
$$

Examining the matrix $\left(u_{\alpha \beta}\right)$ and noting that $i \leq m(k) \leq m / 12$, it is clear that the maximum product is induced when $r=i$ and $u_{r 1}=i^{k}$.

From (3.7) and (3.8), we have

$$
\left|a_{i}\right| \leq\left(\left|\frac{B_{k}}{2 k}\right| 2^{12} \cdots(i-1)^{6(i-1)}\right)^{-1} B\left(U_{k i}\right),
$$

which implies

$$
\left|a_{i}\right| \leq\left|\frac{B_{k}}{2 k}\right|^{-1}\left(\left(2^{12}\right) \cdots(i-1)^{6(i-1)}\right)^{-1} 2^{i-1} i^{k}(1)\left(\left(2^{12}\right) \cdots(i-1)^{6(i-1)}\right)=\left|\frac{2 k}{B_{k}}\right| 2^{i-1} i^{k} .
$$

Thus,

$$
\sum_{j=1}^{m(k)}\left|a_{j}\right| \epsilon^{j} \leq\left|\frac{2 k}{B_{k}}\right| \sum_{j=1}^{m(k)} 2^{j-1} j^{k} \epsilon^{j} .
$$

A standard bound on the Bernoulli numbers (see $\S 15$, [IR], p. 232) gives us $\left|\frac{2 k}{B_{k}}\right|<$ $\frac{k(\pi e)^{k}}{(k / 2)^{k}}$, which implies

$$
\left|\frac{2 k}{B_{k}}\right| \sum_{j=1}^{m(k)} 2^{j-1} j^{k} \epsilon^{j}<\frac{k(\pi e)^{k}}{(k / 2)^{k}} \sum_{j=1}^{m(k)} 2^{j-1} j^{k} \epsilon^{j} .
$$

Noting that $m(k) \leq k / 12$ and $2^{j-1} j^{k} \epsilon^{j}$ is monotonically increasing for $1 \leq j \leq$ $m(k)$, we have

$$
\frac{k(\pi e)^{k}}{(k / 2)^{k}} \sum_{j=1}^{m(k)} 2^{j-1} j^{k} \epsilon^{j}<\frac{k(\pi e)^{k}}{(k / 2)^{k}} m(k)^{k+1} \epsilon^{m(k)} 2^{m(k)-1} \leq \frac{k^{2}\left(\pi e \epsilon^{1 / 12} 2^{1 / 12}\right)^{k}}{24(6)^{k}},
$$


which decreases monotonically as $k \geq 59$ approaches infinity. Furthermore, for $k \geq 300, \frac{k^{2}\left(\pi e \epsilon^{1 / 12} 2^{1 / 12}\right)^{k}}{24(6)^{k}}<\frac{1-\delta}{4}$. This implies (3.2) and completes the proof.

As stated before, if a zero of a modular function lies on $A$, then the $j$-invariant of that zero is a real number in the interval $[0,1728]$. In order to prove Corollary 3 , however, we need more information. Following $[\mathrm{AO}$, we define the following polynomials $h_{k}$ and modular forms $\widetilde{E}_{k}(z)$ for even $k \geq 4$ :

$$
\begin{aligned}
& h_{k}(x):=\left\{\begin{array}{lll}
1 & \text { if } k \equiv 0 \quad(\bmod 12), \\
x^{2}(x-1728) & \text { if } k \equiv 2 \quad(\bmod 12), \\
x & \text { if } k \equiv 4 \quad(\bmod 12), \\
x-1728 & \text { if } k \equiv 6 \quad(\bmod 12), \\
x^{2} & \text { if } k \equiv 8 \quad(\bmod 12), \\
x(x-1728) & \text { if } k \equiv 10 \quad(\bmod 12),
\end{array}\right. \\
& \widetilde{E}_{k}(z):=\left\{\begin{array}{lll}
1 & \text { if } k \equiv 0 \quad(\bmod 12), \\
E_{4}(z)^{2} E_{6}(z) & \text { if } k \equiv 2 \quad(\bmod 12), \\
E_{4}(z) & \text { if } k \equiv 4 \quad(\bmod 12), \\
E_{6}(z) & \text { if } k \equiv 6 \quad(\bmod 12), \\
E_{4}(z)^{2} & \text { if } k \equiv 8 \quad(\bmod 12), \\
E_{4}(z) E_{6}(z) & \text { if } k \equiv 10 \quad(\bmod 12) .
\end{array}\right.
\end{aligned}
$$

In [AO], Ahlgren and Ono prove the following lemma using these definitions:

Lemma 3.2. Suppose that $f \in M_{k}$ has leading coefficient 1 . Let $\widetilde{F}(f, x)$ be the unique rational function in $x$ for which

$$
f(z)=\Delta(z)^{m(k)} \widetilde{E}_{k}(z) \widetilde{F}(f, j(z))
$$

Then $\widetilde{F}(f, x)$ is a polynomial.

For $f(z) \in M_{k}$, we then define the polynomial $F(f, x)$ by

$$
F(f, x):=h_{k}(x) \widetilde{F}(f, x) .
$$

Note that $F$ is constructed so that $F(f, x)=0$ if and only if $x=j(z)$, where $z \in \mathbf{F}$ is a zero of $f$.

Proof of Corollary 3. Fix a weight $k$. We may take as a basis for $M_{k}$ the set of modular forms $\left\{\Delta^{i} E_{4}^{r_{i}} E_{6}^{s_{i}}\right\}_{i=0}^{m(k)}$, where we choose integers $r_{i} \geq 0$ and $1 \geq s_{i} \geq 0$ such that $4 r_{i}+6 s_{i}=k-12 i$. With respect to this basis we may write the gap function $F_{k}$ as

$$
F_{k}=\sum_{j=0}^{m(k)} a_{j}^{\prime} \Delta^{j} E_{4}^{r_{j}} E_{6}^{s_{j}}=1+c(m(k)+1) q^{m(k)+1}+c(m(k)+2) q^{m(k)+2}+\cdots
$$

Observe that the coefficients of the $q$-expansions of $\Delta, E_{4}$, and $E_{6}$ are integers. Therefore, $a_{j}^{\prime}, c_{j} \in \mathbb{Z}$; the coefficients of $F_{k}$ are integers. Noting that $\widetilde{E}_{k}(z)$ is a product of $E_{4}$ and $E_{6}$ for each $k$, we have that $F\left(F_{k}, x\right)$ is a monic polynomial in $\mathbb{Z}[x]$. Thus the zeros of $F\left(F_{k}, x\right)$ are algebraic integers, and these zeros are precisely the $j(z)$ where $F_{k}(z)=0$. 
Consider the polynomial

$$
S_{p}(x):=\prod_{\substack{E / \overline{\mathbb{F}}_{p} \\ \text { supersingular }}}(x-j(E)) \in \mathbb{F}_{p}[x] .
$$

A well-known result of Deligne (see $[\underline{S}]$ ) implies that if $p \geq 5$ is prime, then

$$
S_{p}(x) \equiv F\left(E_{p-1}, x\right) \quad(\bmod p) .
$$

Note that the Von-Staudt congruences imply that $\frac{2(p-1)}{B_{p-1}} \equiv 0(\bmod p)$. In addition, $F_{k}$, when considered as a modular form of weight $k$ modulo $p$, is determined by its first $m(k)+1$ Fourier coefficients. Thus we have the following congruence of $q$-expansion coefficients:

$$
F_{p-1} \equiv E_{p-1} \equiv 1 \quad(\bmod p)
$$

This implies

$$
F\left(F_{p-1}, x\right) \equiv F\left(E_{p-1}, x\right) \equiv S_{p}(x) \quad(\bmod p)
$$

\section{ACKNOWLEDGMENTS}

The author is grateful to K. Ono for organizing this work as an undergraduate summer research opportunity. M. Baker and W. Stein also deserve thanks for their help with preparatory reading and editing, respectively.

\section{REFERENCES}

[AO] S. Ahlgren and K. Ono, Weierstrass points on $X_{0}(p)$ and supersingular $j$-invariants, Math. Ann. 325 (2003), 355-368. MR 2004b:11086

[A] T. M. Apostol, Introduction to Analytic Number Theory, Springer-Verlag, New York, 1976. MR 55:7892

[IR] K. Ireland and M. Rosen, A Classical Introduction to Modern Number Theory, Graduate Texts in Mathematics, no. 84, Springer-Verlag, New York, 1990. MR 92e:11001

[K] N. Koblitz, Introduction to Elliptic Curves and Modular Forms, Graduate Texts in Mathematics, no. 97, Springer-Verlag, New York, 1993. MR 94a:11078

[MOS] C. L. Mallows, A. M. Odlyzko, and N. J. A. Sloane, Upper Bounds for Modular Forms, Lattices, and Codes, Journal of Algebra 36 (1975), 68-76. MR 51:12711

[RSD] F. K. C. Rankin and H. P. F. Swinnerton-Dyer, On the zeros of Eisenstein series, Bull. London Math. Soc. 2 (1970), 169-170. MR 41:5298

[S] J.-P. Serre, Congruences et formes modulaires (d'après H. P. F. Swinnerton-Dyer), Séminaire Bourbaki 416 (1971-1972), 319-338. MR 57:5904a

4404 South Avenue West, Missoula, Montana 59804

E-mail address: getz@fas.harvard.edu 